\title{
ON FUNCTIONS WITH THE CAUCHY DIFFERENCE BOUNDED BY A FUNCTIONAL. PART II
}

WŁODZIMIERZ FECHNER

Received 10 December 2004 and in revised form 17 May 2005

We are going to consider the functional inequality $f(x+y)-f(x)-f(y) \geq \phi(x, y)$, $x, y \in X$, where $(X,+)$ is an abelian group, and $\phi: X \times X \rightarrow \mathbb{R}$ and $f: X \rightarrow \mathbb{R}$ are unknown mappings. In particular, we will give conditions which force biadditivity and symmetry of $\phi$ and the representation $f(x)=(1 / 2) \phi(x, x)+a(x)$ for $x \in X$, where $a$ is an additive function. In the present paper, we continue and develop our earlier studies published by the author (2004).

Let $(X,+)$ be an abelian group. We consider the functional inequality

$$
f(x+y)-f(x)-f(y) \geq \phi(x, y), \quad x, y \in X,
$$

where $\phi: X \times X \rightarrow \mathbb{R}$ and $f: X \rightarrow \mathbb{R}$ are unknown mappings.

First, we quote [3, Proposition].

Proposition 1. If $f: X \rightarrow \mathbb{R}, \phi: X \times X \rightarrow \mathbb{R}$ satisfy (1) and

$$
\phi(x,-x) \geq-\phi(x, x), \quad x \in X
$$

then, (a) $f(0) \leq 0$; (b) $f(x)+f(-x) \leq \phi(x, x)$ for $x \in X$; (c) $f(2 x) \geq 3 f(x)+f(-x)$ for $x \in X$.

One can see that an even function $f: X \rightarrow \mathbb{R}$ which fulfills assumptions of Proposition 1 satisfies $f(2 x) \geq 4 f(x)$ for $x \in X$. This observation was used in [3], where a new function $Q: X \rightarrow \mathbb{R}$ was defined by the formula $Q(x):=\lim _{k \rightarrow+\infty} f\left(2^{k} x\right) / 4^{k}$ for $x \in X$. The resulted equality $Q(2 x)=4 Q(x)$ for $x \in X$ played a crucial role.

The main idea of the present paper is to drop the assumption that $f$ is even and use Proposition 1 (c) to get a limit function $\varphi: X \rightarrow \mathbb{R}$ satisfying the equality $\varphi(2 x)=3 \varphi(x)+$ $\varphi(-x)$ for $x \in X$ (see Theorems 14 and 16). 
It is assumed that $\mathbb{N}=\{1,2, \ldots\}$ and $\mathbb{N}_{0}=\{0,1,2, \ldots\}$.

Let us quote here [3, Lemma 1].

Lemma 2. Assume that $f: X \rightarrow \mathbb{R}$ and $\phi: X \times X \rightarrow \mathbb{R}$ satisfy (1). If

$$
\begin{gathered}
\phi(x,-y) \geq-\phi(x, y), \quad x, y \in X, \\
f(2 x) \leq 4 f(x), \quad x \in X,
\end{gathered}
$$

then

$$
f(x)=\frac{1}{2} \phi(x, x), \quad x \in X
$$

Moreover, $\phi$ is biadditive and symmetric.

The foregoing result was the main tool in [3]. In fact, this lemma, in slightly different version, was first proved by K. Baron (see [4]). In the present paper, we need to state a more general lemma, which works for maps satisfying $f(2 x) \leq 3 f(x)+f(-x)$ for $x \in X$.

Lemma 3. Assume that $f: X \rightarrow \mathbb{R}$ and $\phi: X \times X \rightarrow \mathbb{R}$ satisfy (1) and (3). If

$$
f(2 x) \leq 3 f(x)+f(-x), \quad x \in X,
$$

then there exists an additive function $a: X \rightarrow \mathbb{R}$ such that

$$
f(x)=\frac{1}{2} \phi(x, x)+a(x), \quad x \in X .
$$

Moreover, $\phi$ is biadditive and symmetric.

Proof. Setting $-y$ instead of $y$ in (1), we obtain

$$
f(x-y)-f(x)-f(-y) \geq \phi(x,-y) \geq-\phi(x, y), \quad x, y \in X .
$$

Adding this to (1) leads to

$$
f(x+y)+f(x-y) \geq 2 f(x)+f(y)+f(-y), \quad x, y \in X .
$$

Fix arbitrarily $u, v \in X$. Applying this inequality with $x=u+v$ and $y=u-v$ and using (6), we infer that

$$
\begin{aligned}
3 f(u) & +f(-u)+3 f(v)+f(-v) \\
\geq & f(2 u)+f(2 v) \geq 2 f(u+v)+f(u-v)+f(v-u), \quad u, v \in X .
\end{aligned}
$$

The last two inequalities imply that $f$ satisfies the equality

$$
f(x+y)+f(x-y)=2 f(x)+f(y)+f(-y), \quad x, y \in X .
$$

Now, define $q: X \rightarrow \mathbb{R}$ and $a: X \rightarrow \mathbb{R}$ by the formulas

$$
a(x):=\frac{f(x)-f(-x)}{2}, \quad q(x):=\frac{f(x)+f(-x)}{2}, \quad x \in X .
$$


It is clear that

$$
a(x+y)+a(x-y)=2 a(x), \quad x, y \in X,
$$

thus $a$ is additive. Moreover,

$$
q(x+y)+q(x-y)=2 q(x)+2 q(y), \quad x, y \in X,
$$

that is, $q$ is quadratic. There exists a biadditive and symmetric functional $B: X \times X \rightarrow \mathbb{R}$ such that $q(x)=B(x, x)$ for $x \in X$ (see, e.g., Aczél and Dhombres [1, Chapter 11, Proposition 1]). Moreover, we have

$$
q(x+y)-q(x)-q(y)=2 B(x, y), \quad x, y \in X .
$$

This implies that $2 B(x, y) \geq \phi(x, y)$ for $x, y \in X$. By the use of this, (3), and the biadditivity of $B$, we get that $\phi(x, y) \geq-\phi(x,-y) \geq-2 B(x,-y)=2 B(x, y)$ for $x, y \in X$. So $2 B=\phi$. This completes the proof.

Our next step is to drop the assumption of the evenness of function $f$ in $[3$, Lemma 3]. We have the following generalization of this result.

Recall that a group $X$ is called uniquely 2-divisible if and only if the map $X \ni x \rightarrow$ $x+x \in X$ is bijective.

Lemma 4. Assume $X$ to be uniquely 2-divisible, $f: X \rightarrow \mathbb{R}, \phi: X \times X \rightarrow \mathbb{R}$ satisfy (1), (2), and

$$
\phi(2 x, 2 x) \leq 4 \phi(x, x), \quad x \in X .
$$

If $f$ is nonnegative, then $f$ is even and $f(x)=(1 / 2) \phi(x, x)$ for $x \in X$.

Proof. By Proposition 1(c) and nonnegativity of $f$, we get that for $x \in X$, the sequence $\left(2^{n} f\left(x / 2^{n}\right)\right)_{n \in \mathbb{N}}$ is nonincreasing and nonnegative and thus convergent. So, the formula

$$
A(x):=\lim _{n \rightarrow+\infty} 2^{n} f\left(\frac{x}{2^{n}}\right), \quad x \in X,
$$

correctly defines a map $A: X \rightarrow \mathbb{R}$. Moreover, $A(x) \geq 0$ and $A(2 x)=2 A(x)$ for $x \in X$.

Proposition $1(\mathrm{c})$ implies that

$$
2^{n} f\left(\frac{x}{2^{n-1}}\right) \geq 3 \cdot 2^{n} f\left(\frac{x}{2^{n}}\right)+2^{n} f\left(\frac{-x}{2^{n}}\right), \quad x \in X, n \in \mathbb{N} .
$$

So

$$
2 A(x)=A(2 x) \geq 3 A(x)+A(-x), \quad x \in X,
$$

and we can easily observe that $A=0$.

Now, we will follow the original proof of [3, Lemma 3]. Fix an $x \in X$. From (1) and (16), we derive inductively the estimations

$$
2^{k} f\left(\frac{x}{2^{k-1}}\right)-2^{k+1} f\left(\frac{x}{2^{k}}\right) \geq 2^{k} \phi\left(\frac{x}{2^{k}}, \frac{x}{2^{k}}\right) \geq \frac{1}{2^{k}} \phi(x, x),
$$


for all $k \in \mathbb{N}$. Summing up these inequalities side by side for $k \in\{1, \ldots, n\}$, we get that

$$
2 f(x)-2^{n+1} f\left(\frac{x}{2^{n}}\right) \geq \sum_{k=1}^{n} \frac{1}{2^{k}} \phi(x, x), \quad n \in \mathbb{N} .
$$

Letting $n$ tend to $+\infty$ yields the inequality $2 f(x) \geq \phi(x, x)$.

On the other hand, Proposition 1 (b) states that $f(x)+f(-x) \leq \phi(x, x)$ for $x \in X$. So, $f$ is even and $f(x)=(1 / 2) \phi(x, x)$ for $x \in X$. This completes the proof.

In the next lemma, we will provide a certain property of the inequality from Proposition 1(c).

Lemma 5. Assume $X$ to be uniquely 2-divisible. If $f: X \rightarrow \mathbb{R}$ satisfies

$$
\begin{gathered}
f(2 x) \geq 3 f(x)+f(-x), \quad x \in X, \\
\forall_{x \in X} \exists_{k_{0} \in \mathbb{N}} \forall_{k \geq k_{0}} f\left(\frac{x}{2^{k}}\right) \geq 0,
\end{gathered}
$$

then $f \geq 0$.

Proof. Define a sequence $\left(\varphi_{k}\right)_{k \in \mathbb{N}_{0}}$ of real mappings on $X$ by the formula

$$
\varphi_{k}(x):=\frac{4^{k}+2^{k}}{2} f\left(\frac{x}{2^{k}}\right)+\frac{4^{k}-2^{k}}{2} f\left(-\frac{x}{2^{k}}\right), \quad x \in X, k \in \mathbb{N}_{0} .
$$

We will show that this sequence is nonincreasing. Fix an $x \in X$ and $k \in \mathbb{N}_{0}$. We have

$$
\begin{aligned}
\varphi_{k}(x)= & \frac{4^{k}+2^{k}}{2} f\left(\frac{x}{2^{k}}\right)+\frac{4^{k}-2^{k}}{2} f\left(-\frac{x}{2^{k}}\right) \\
\geq & \frac{4^{k}+2^{k}}{2}\left[3 f\left(\frac{x}{2^{k+1}}\right)+f\left(-\frac{x}{2^{k+1}}\right)\right] \\
& +\frac{4^{k}-2^{k}}{2}\left[3 f\left(-\frac{x}{2^{k+1}}\right)+f\left(\frac{x}{2^{k+1}}\right)\right] \\
= & \frac{4^{k+1}+2^{k+1}}{2} f\left(\frac{x}{2^{k+1}}\right)+\frac{4^{k+1}-2^{k+1}}{2} f\left(-\frac{x}{2^{k+1}}\right) \\
= & \varphi_{k+1}(x) .
\end{aligned}
$$

The assumption (23) implies that the sequence $\left(\varphi_{k}(x)\right)_{k \in \mathbb{N}_{0}}$ is nonnegative for $x \in X$. In particular $f(x)=\varphi_{0}(x) \geq 0$ for $x \in X$. This completes the proof.

Now, we may join this lemma with our Lemmas 4, 2 and Proposition 1(c) to get the following result.

Corollary 6. Assume $X$ to be uniquely 2-divisible, $f: X \rightarrow \mathbb{R}, \phi: X \times X \rightarrow \mathbb{R}$ satisfy (1), (2), (16), and (23). Then $f$ is nonnegative, even, and $f(x)=(1 / 2) \phi(x, x)$ for $x \in X$. Moreover, if (3) is also satisfied, then $\phi$ is biadditive and symmetric. 
Next, we will quote [3, Theorem 2].

Theorem 7. Assume $X$ to be uniquely 2-divisible and that $f: X \rightarrow \mathbb{R}, \phi: X \times X \rightarrow \mathbb{R}$ satisfy (1), (3), (16) jointly with

$$
f(x)+f(-x) \geq 0, \quad x \in X .
$$

Then there exists an additive function $a: X \rightarrow \mathbb{R}$ such that

$$
f(x)=\frac{1}{2} \phi(x, x)+a(x), \quad x \in X .
$$

Moreover, $\phi$ is biadditive and symmetric.

This result together with Lemma 5 applied for a map $x \mapsto f(x)+f(-x)$ leads to the following corollary.

Corollary 8. Assume $X$ to be uniquely 2-divisible and that $f: X \rightarrow \mathbb{R}, \phi: X \times X \rightarrow \mathbb{R}$ satisfy (1), (3), (16) jointly with

$$
\forall_{x \in X} \exists_{k_{0} \in \mathbb{N}} \forall_{k \geq k_{0}} f\left(\frac{x}{2^{k}}\right)+f\left(-\frac{x}{2^{k}}\right) \geq 0 .
$$

Then there exists an additive function $a: X \rightarrow \mathbb{R}$ such that

$$
f(x)=\frac{1}{2} \phi(x, x)+a(x), \quad x \in X .
$$

Moreover, $\phi$ is biadditive and symmetric.

Now, we quote [2, Corollary 2].

Corollary 9. Assume $X$ to be a real linear space and that $f: X \rightarrow \mathbb{R}, \phi: X \times X \rightarrow \mathbb{R}$ satisfy (1), $f$ is nonnegative, and $\phi(x, \cdot)$ is homogeneous for $x \in X$. Then $\phi$ is bilinear and symmetric and $f(x)=(1 / 2) \phi(x, x)$ for $x \in X$.

In the light of Lemma 5, we get then the following corollary.

Corollary 10. Assume $X$ to be a real linear space, $f: X \rightarrow \mathbb{R}, \phi: X \times X \rightarrow \mathbb{R}$ satisfy (1), (23), and $\phi(x, \cdot)$ is homogeneous for $x \in X$. Then $\phi$ is bilinear and symmetric and $f(x)=$ $(1 / 2) \phi(x, x) \geq 0$ for $x \in X$.

We recall also the following corollary.

Corollary $11[2$, Corollary 1$]$. Assume $X$ to be a real linear space and that $f: X \rightarrow \mathbb{R}$, $\phi: X \times X \rightarrow \mathbb{R}$ satisfy (1). If for every $x \in X$ the function $\mathbb{R} \ni t \mapsto f(t x) \in \mathbb{R}$ has the property that its Jensen convexity implies its convexity and $f$ satisfies $(26)$ with $\phi(x, \cdot)$ being homogeneous for $x \in X$, then there exists a linear functional $L: X \rightarrow \mathbb{R}$ such that

$$
f(x)=\frac{1}{2} \phi(x, x)+L(x), \quad x \in X .
$$

Moreover, $\phi$ is bilinear and symmetric. 
A similar reasoning as above allows us to derive the following fact.

Corollary 12. Assume $X$ to be a real linear space and that $f: X \rightarrow \mathbb{R}, \phi: X \times X \rightarrow \mathbb{R}$ satisfy (1). If for every $x \in X$ the function $\mathbb{R} \ni t \mapsto f(t x) \in \mathbb{R}$ has the property that its Jensen convexity implies its convexity and $f$ satisfies $(28)$ with $\phi(x, \cdot)$ being homogeneous for $x \in X$, then there exists a linear functional $L: X \rightarrow \mathbb{R}$ such that

$$
f(x)=\frac{1}{2} \phi(x, x)+L(x), \quad x \in X .
$$

Moreover, $\phi$ is bilinear and symmetric.

Remark 13. If $X$ is a real linear topological Hausdorff space, then (23) is satisfied if $f$ is nonnegative in a certain neighborhood of zero.

Now, we state and prove our next result.

Theorem 14. Assume $X$ to be uniquely 2-divisible, $f: X \rightarrow \mathbb{R}, \phi: X \times X \rightarrow \mathbb{R}$ satisfy (1), (3),

$$
\begin{gathered}
\phi(2 x, 2 y) \leq 4 \phi(x, y), \quad x, y \in X, \\
\forall_{x \in X}\left(\liminf _{k \rightarrow+\infty}\left[4^{k} f\left(\frac{x}{2^{k}}\right)+4^{k} f\left(\frac{-x}{2^{k}}\right)\right]>-\infty\right), \\
\forall_{x \in X}\left(\liminf _{k \rightarrow+\infty} 2^{k} f\left(\frac{x}{2^{k}}\right)>-\infty \vee \limsup _{k \rightarrow+\infty} 2^{k} f\left(\frac{-x}{2^{k}}\right)<+\infty\right) .
\end{gathered}
$$

Then there exists an additive function $a: X \rightarrow \mathbb{R}$ such that

$$
f(x)=\frac{1}{2} \phi(x, x)+a(x), \quad x \in X .
$$

Moreover, $\phi$ is biadditive and symmetric.

Proof. Define a sequence $\left(\varphi_{k}\right)_{k \in \mathbb{N}_{0}}$ of real mappings on $X$ by the formula (24). We have already checked (proof of Lemma 5) that this sequence is nonincreasing. We will show that it is pointwise bounded. Fix an $x \in X$ and observe that

$$
\begin{array}{ll}
\varphi_{k}(x)=\frac{4^{k}+2^{k}}{2 \cdot 4^{k}}\left[4^{k} f\left(\frac{x}{2^{k}}\right)+4^{k} f\left(\frac{-x}{2^{k}}\right)\right]-2^{k} f\left(\frac{-x}{2^{k}}\right), \quad k \in \mathbb{N}_{0}, \\
\varphi_{k}(x)=\frac{4^{k}-2^{k}}{2 \cdot 4^{k}}\left[4^{k} f\left(\frac{x}{2^{k}}\right)+4^{k} f\left(\frac{-x}{2^{k}}\right)\right]+2^{k} f\left(\frac{x}{2^{k}}\right), \quad k \in \mathbb{N}_{0} .
\end{array}
$$

So, by (33), the sequence $\left(\varphi_{k}\right)_{k \in \mathbb{N}_{0}}$ is pointwise convergent. Define $\varphi: X \rightarrow \mathbb{R}$ by $\varphi(x):=$ $\lim _{k \rightarrow+\infty} \varphi_{k}(x)$ for $x \in X$. Observe that

$$
\varphi_{k+1}(2 x)=3 \varphi_{k}(x)+\varphi_{k}(-x), \quad x \in X, k \in \mathbb{N}_{0},
$$

and thus

$$
\varphi(2 x)=3 \varphi(x)+\varphi(-x), \quad x \in X .
$$


Next, by the definition of $\varphi$ and $\varphi_{k},(1)$ and (32), we have

$$
\begin{aligned}
\varphi(x+ & y)-\varphi(x)-\varphi(y)=\lim _{k \rightarrow+\infty}\left[\varphi_{k}(x+y)-\varphi_{k}(x)-\varphi_{k}(y)\right] \\
& \geq \limsup _{k \rightarrow+\infty} \frac{4^{k}+2^{k}}{2} \phi\left(\frac{x}{2^{k}}, \frac{y}{2^{k}}\right)+\limsup _{k \rightarrow+\infty} \frac{4^{k}-2^{k}}{2} \phi\left(\frac{-x}{2^{k}}, \frac{-y}{2^{k}}\right) \\
& \geq \frac{1}{2} \phi(x, y)+\frac{1}{2} \phi(-x,-y), \quad x, y \in X .
\end{aligned}
$$

Define $\phi_{1}: X \times X \rightarrow \mathbb{R}$ by $\phi_{1}(x, y):=(1 / 2)[\phi(x, y)+\phi(-x,-y)]$ for $x, y \in X$. Now, we may apply Lemma 3 with $\varphi$ and $\phi_{1}$ to get that $\phi_{1}$ is biadditive and symmetric and $\varphi=$ $q+a$, where $q$ is a quadratic mapping and $a$ is an additive one. Moreover,

$$
\varphi(x+y)-\varphi(x)-\varphi(y)=q(x+y)-q(x)-q(y)=\phi_{1}(x, y), \quad x, y \in X .
$$

Now, put $f_{1}:=f-\varphi$ and $\phi_{2}:=\phi-\phi_{1}$. We have $f_{1} \geq 0$ and

$$
f_{1}(x+y)-f_{1}(x)-f_{1}(y) \geq \phi_{2}(x, y), \quad x, y \in X .
$$

Lemma 4 applied for $f=f_{1}$ and $\phi=\phi_{2}$ implies that $f_{1}$ is even and $f_{1}(2 x)=4 f_{1}(x)$ for $x \in X$. By Proposition 1(c), we have

$$
\begin{aligned}
3 \varphi(x) & +\varphi(-x)+4 f_{1}(x)=\varphi(2 x)+f_{1}(2 x)=f(2 x) \\
\geq & 3 f(x)+f(-x)=3 \varphi(x)+\varphi(-x)+4 f_{1}(x), \quad x \in X .
\end{aligned}
$$

So $f(2 x)=3 f(x)+f(-x)$ for $x \in X$. This means that $f=\varphi$, and as a consequence $\phi_{2}=0$. This completes the proof.

Remark 15. The assumption (33) is fulfilled if $f$ satisfies the condition (26), which appears (among others) in Theorem 7. But Theorem 14 does not generalize Theorem 7 or Corollary 8 , unless we are able to replace the assumption (32) by (16) in Theorem 14 (note that (32) in its whole strength was used only to prove that $\varphi(x+y)-\varphi(x)-\varphi(y) \geq$ $\phi_{1}(x, y)$ for $\left.x, y \in X\right)$.

Now, we will state and prove our last result, which yields a generalization to [3, Theorem 1].

Theorem 16. Assume that $f: X \rightarrow \mathbb{R}$ and $\phi: X \times X \rightarrow \mathbb{R}$ satisfy (1), (3) and

$$
\begin{gathered}
\limsup _{k \rightarrow+\infty} \frac{1}{4^{k}} \phi\left(2^{k} x, 2^{k} x\right)<+\infty, \quad x \in X, \\
\liminf _{k \rightarrow+\infty} \frac{1}{4^{k}} \phi\left(2^{k} x, 2^{k} y\right) \geq \phi(x, y), \quad x, y \in X .
\end{gathered}
$$

If the sequence $\left(2^{-k}\left[f\left(2^{k} x\right)-f\left(-2^{k} x\right)\right]\right)_{k \in \mathbb{N}}$ is pointwise convergent to a superadditive function, then there exists a subadditive function $A: X \rightarrow \mathbb{R}$ such that

$$
f(x)=\frac{1}{2} \phi(x, x)-A(x), \quad x \in X .
$$

Moreover, $\phi$ is biadditive and symmetric. 
Proof. Define a sequence $\left(\hat{\varphi}_{k}\right)_{k \in \mathbb{N}_{0}}$ of real mappings on $X$ by the formula

$$
\hat{\varphi}_{k}(x):=\frac{4^{-k}+2^{-k}}{2} f\left(2^{k} x\right)+\frac{4^{-k}-2^{-k}}{2} f\left(-2^{k} x\right), \quad x \in X, k \in \mathbb{N}_{0} .
$$

We will show that this sequence is convergent. Fix an $x \in X$. We have

$$
\hat{\varphi}_{k}(x)=\frac{f\left(2^{k} x\right)+f\left(-2^{k} x\right)}{2 \cdot 4^{k}}+\frac{f\left(2^{k} x\right)-f\left(-2^{k} x\right)}{2^{k+1}}, \quad k \in \mathbb{N}_{0} .
$$

Observe that by Proposition 1(c), the first summand is nondecreasing and (by Proposition 1(b)) pointwise upper bounded by $4^{-k} \phi\left(2^{k} x, 2^{k} x\right)$, whereas the second one is convergent by the assumption. Thus the sequence $\left(\hat{\varphi}_{k}\right)_{k \in \mathbb{N}}$ is convergent. Therefore, the formula

$$
\hat{\varphi}(x):=\lim _{k \rightarrow+\infty} \hat{\varphi}_{k}(x), \quad x \in X,
$$

correctly defines a map $\hat{\varphi}: X \rightarrow \mathbb{R}$. Moreover, $\hat{\varphi}(2 x)=3 \hat{\varphi}(x)+\hat{\varphi}(-x)$ for $x \in X$ and the following inequality is satisfied:

$$
\begin{aligned}
\hat{\varphi}(x+y) & -\hat{\varphi}(x)-\hat{\varphi}(y) \\
= & \lim _{k \rightarrow+\infty} \frac{1}{2} \cdot 4^{-k}\left[f\left(2^{k} x+2^{k} y\right)-f\left(2^{k} x\right)-f\left(2^{k} y\right)\right] \\
& +\frac{1}{2} \cdot 4^{-k}\left[f\left(-2^{k} x-2^{k} y\right)-f\left(-2^{k} x\right)-f\left(-2^{k} y\right)\right] \\
& +2^{-k-1}\left[f\left(2^{k} x+2^{k} y\right)-f\left(2^{k} x\right)-f\left(2^{k} y\right)\right] \\
& -2^{-k-1}\left[f\left(-2^{k} x-2^{k} y\right)-f\left(-2^{k} x\right)-f\left(-2^{k} y\right)\right] \\
\geq & \liminf _{k \rightarrow+\infty} \frac{1}{2} \cdot 4^{-k}\left[\phi\left(2^{k} x, 2^{k} y\right)+\phi\left(-2^{k} x,-2^{k} y\right)\right] \\
& +\frac{1}{2}[p(x+y)-p(x)-p(y)] \\
\geq & \frac{1}{2}[\phi(x, y)+\phi(-x,-y)], \quad x, y \in X
\end{aligned}
$$

where $p: X \rightarrow \mathbb{R}$ is defined by

$$
p(x):=\lim _{k \rightarrow+\infty} \frac{1}{2^{k}}\left[f\left(2^{k} x\right)-f\left(-2^{k} x\right)\right], \quad x \in X .
$$

Lemma 3 states that the map $\phi_{1}: X \times X \rightarrow \mathbb{R}$, defined by $\phi_{1}(x, y)=(1 / 2)[\phi(x, y)+$ $\phi(-x,-y)]$ for $x, y \in X$, is biadditive and symmetric and $\hat{\varphi}(x)=(1 / 2) \phi_{1}(x, x)+a(x)$ for $x \in X$, where $a$ is an additive mapping. It implies that

$$
\hat{\varphi}(x+y)-\hat{\varphi}(x)-\hat{\varphi}(y)=\phi_{1}(x, y), \quad x, y \in X,
$$

that is, the foregoing estimation holds with the equality. In particular,

$$
\lim _{k \rightarrow+\infty} 4^{-k}\left[f\left(2^{k} x+2^{k} y\right)-f\left(2^{k} x\right)-f\left(2^{k} y\right)\right]=\phi(x, y), \quad x, y \in X .
$$


Moreover, observe that $\hat{\varphi}_{k}(x)-\hat{\varphi}_{k}(-x)=\left(1 / 2^{k}\right)\left(f\left(2^{k} x\right)-f\left(-2^{k} x\right)\right)$ for $x \in X$ and $k \in$ $\mathbb{N}_{0}$, whence $2 a=p$.

Now, put $f_{1}:=f-\hat{\varphi}$ and $\phi_{2}:=\phi-\phi_{1}$. Clearly, $\phi_{2}$ satisfies (3), (42), and

$$
f_{1}(x+y)-f_{1}(x)-f_{1}(y) \geq \phi_{2}(x, y), \quad x, y \in X .
$$

Moreover, one has

$$
\begin{aligned}
\lim _{k \rightarrow+\infty} 4^{-k}\left[f_{1}\left(2^{k} x+2^{k} y\right)-f_{1}\left(2^{k} x\right)-f_{1}\left(2^{k} y\right)\right]=\phi_{2}(x, y), \quad x, y \in X, \\
\lim _{k \rightarrow+\infty} \frac{1}{2^{k}}\left[f_{1}\left(2^{k} x\right)-f_{1}\left(-2^{k} x\right)\right] \\
\quad=\lim _{k \rightarrow+\infty} \frac{1}{2^{k}}\left[f\left(2^{k} x\right)-f\left(-2^{k} x\right)\right]-\lim _{k \rightarrow+\infty} \frac{1}{2^{k}}\left[\hat{\varphi}\left(2^{k} x\right)-\hat{\varphi}\left(-2^{k} x\right)\right] \\
\quad=p(x)-2 a(x)=0, \quad x \in X .
\end{aligned}
$$

Split $f_{1}$ into its even and odd parts, that is, define $P, g: X \rightarrow \mathbb{R}$ by $P(x):=(1 / 2)\left[f_{1}(x)+\right.$ $\left.f_{1}(-x)\right]$ and $g(x):=(1 / 2)\left[f_{1}(x)-f_{1}(-x)\right]$ for $x \in X$. Next, fix $x, y \in X$ and apply (51) twice: for $x$ and $y$ and then for $-x$ and $-y$. Summing up side by side the two inequalities obtained and using the definition of $\phi_{1}$ and $\phi_{2}$, we get

$$
f_{1}(x+y)+f_{1}(-x-y)-f_{1}(x)-f_{1}(-x)-f_{1}(y)-f_{1}(-y) \geq 0,
$$

that is, $P$ is superadditive. In particular, due to its evenness, $P$ is nonpositive and $P(2 x) \geq$ $2 P(x)$ for $x \in X$. Thus, the sequence $\left(2^{-k} P\left(2^{k} x\right)\right)_{k \in \mathbb{N}}$ is convergent, whence

$$
\lim _{k \rightarrow+\infty} 4^{-k} P\left(2^{k} x\right)=0, \quad x \in X
$$

This, jointly with (52), implies that

$$
\lim _{k \rightarrow+\infty} 4^{-k}\left[g\left(2^{k} x+2^{k} y\right)-g\left(2^{k} x\right)-g\left(2^{k} y\right)\right]=\phi_{2}(x, y), \quad x, y \in X .
$$

On the other hand, we have

$$
\lim _{k \rightarrow+\infty} 2^{-k} g\left(2^{k} x\right)=\lim _{k \rightarrow+\infty} \frac{1}{2^{k+1}}\left[f_{1}\left(2^{k} x\right)-f_{1}\left(-2^{k} x\right)\right]=0, \quad x \in X
$$

From the last two equalities, it follows that $\phi_{2}=0$. So $\phi=\phi_{1}$ is biadditive and symmetric. It remains to define $A: X \rightarrow \mathbb{R}$ by $A(x):=(1 / 2) \phi(x, x)-f(x)$ for $x \in X$. This completes the proof.

Remark 17. The convergence assumption spoken of in Theorem 16 is weaker than the supposition of the evenness of $f$, used in [3, Theorem 1]. However, we do not know definitely whether or not it could be omitted. 


\section{Cauchy difference}

\section{References}

[1] J. Aczél and J. Dhombres, Functional Equations in Several Variables, Encyclopedia of Mathematics and Its Applications, vol. 31, Cambridge University Press, Cambridge, 1989.

[2] K. Baron and Z. Kominek, On functionals with the Cauchy difference bounded by a homogeneous functional, Bull. Polish Acad. Sci. Math. 51 (2003), no. 3, 301-307.

[3] W. Fechner, On functions with the Cauchy difference bounded by a functional, Bull. Polish Acad. Sci. Math. 52 (2004), no. 3, 265-271.

[4] S. Rolewicz, Ф-convex functions defined on metric spaces, J. Math. Sci. (New York) 115 (2003), no. $5,2631-2652$.

Włodzimierz Fechner: Institute of Mathematics, Faculty of Mathematics, Physics and Chemistry, University of Silesia, 14 Bankowa Street, 40-007 Katowice, Poland

E-mail address: fechner@ux2.math.us.edu.pl 


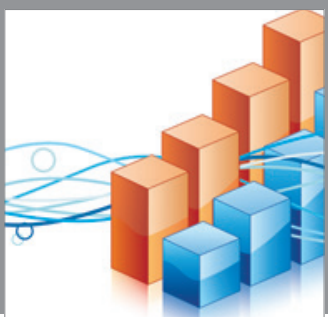

Advances in

Operations Research

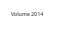

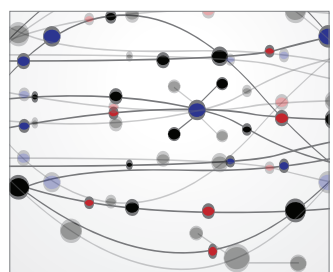

\section{The Scientific} World Journal
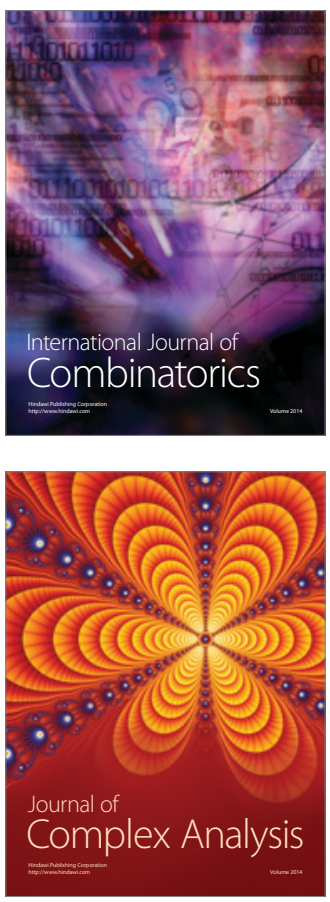

International Journal of

Mathematics and

Mathematical

Sciences
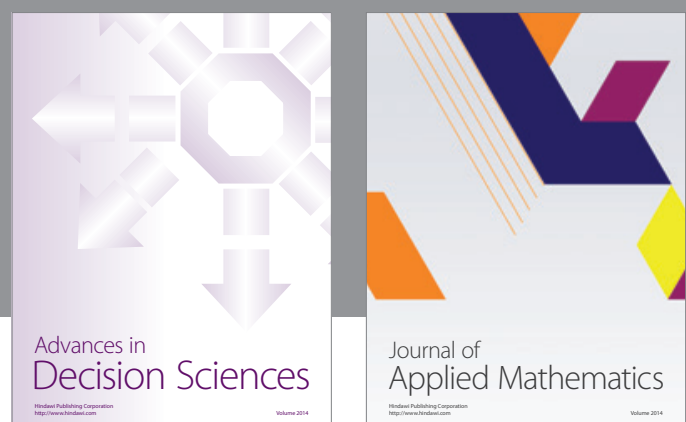

Journal of

Applied Mathematics
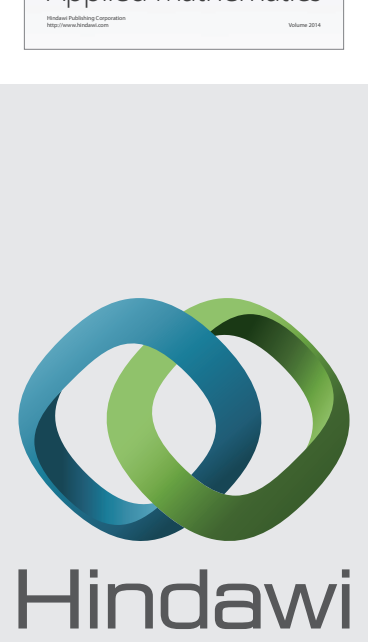

Submit your manuscripts at http://www.hindawi.com
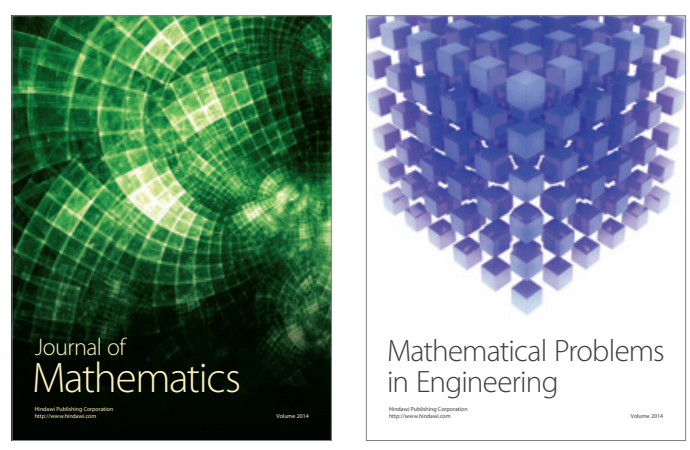

Mathematical Problems in Engineering
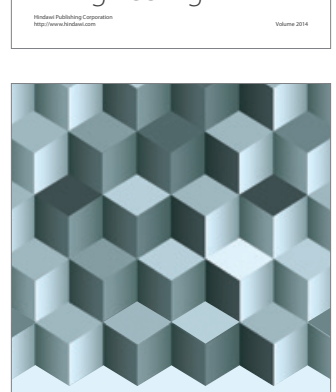

Journal of

Function Spaces
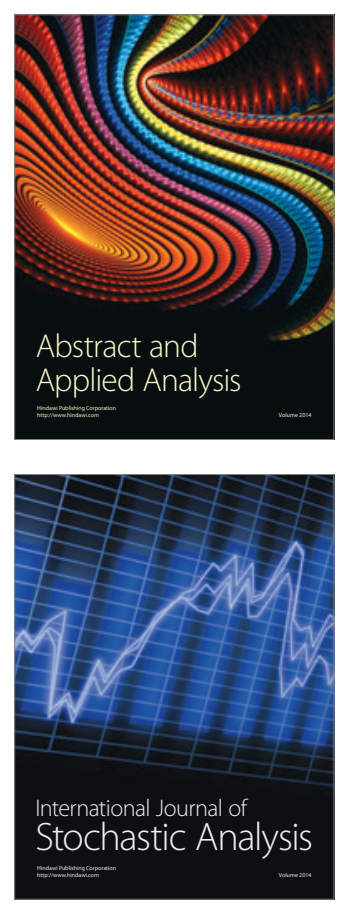

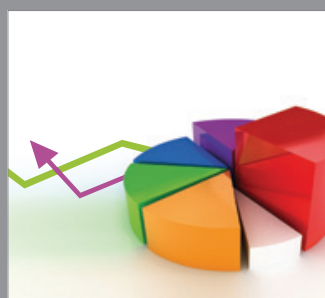

ournal of

Probability and Statistics

Promensencen
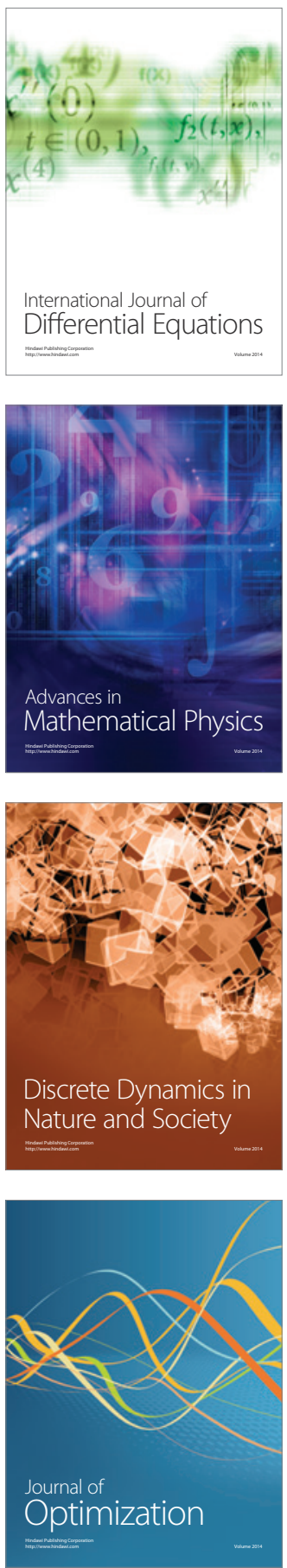